\title{
Impact of blood-brain barrier disruption on newly diagnosed neuromyelitis optica spectrum disorder symptoms and prognosis
}

\author{
Shuolin Liang', Qiuhong Qin ${ }^{2}$, Yulan Tang ${ }^{1}$, Weijing Liao ${ }^{3}$, Yi Yang ${ }^{1}$, Jinrong He ${ }^{1}$, Liangsheng Li $^{1}$ \\ ${ }^{1}$ Department of Neurology, The First Affiliated Hospital, Guangxi Medical University, Nanning 530021, China; ${ }^{2}$ Department of Histology, Guangxi \\ Medical University, Nanning 530021, China; ${ }^{3}$ Department of Neurology, Affiliated Wuming Hospital, Guangxi Medical University, Nanning \\ 530199, China \\ Contributions: (I) Conception and design: S Liang; (II) Administrative support: Y Tang; (III) Provision of study materials or patients: J He, L Li; (IV) \\ Collection and assembly of data: Y Yang; (V) Data analysis and interpretation: Q Qin; (VI) Manuscript writing: All authors; (VII) Final approval of \\ manuscript: All authors. \\ Correspondence to: Yulan Tang. Department of Neurology, The First Affiliated Hospital, Guangxi Medical University, Nanning 530021, China. \\ Email: 13978675500@163.com.
}

Background: Blood-brain barrier (BBB) disruption and ensuing immune activation are central to the pathogenesis of central nervous system (CNS) inflammatory diseases. However, the influence of BBB permeability on the clinical signs and prognosis of newly diagnosed neuromyelitis optica spectrum disorder (NMOSD) has not been examined. We investigate the relationships between BBB permeability as showed by the albumin quotient (qalb) and clinical features of NMOSD.

Methods: Demographic and clinical data of 46 patients, including peripheral blood $(\mathrm{PB})$ measures (serum albumin concentration and total leukocyte, neutrophil, total lymphocyte, CD4+ T cell, and CD8+ T cell counts, complement $\mathrm{C} 3$ and $\mathrm{C} 4$ concentrations, AQP4-IgG titer), autoimmune antibody titers (ANA/SSA/ SSB/Ro-52), and cerebrospinal fluid (CSF) parameters (total leukocyte count, total protein and albumin concentrations, AQP4-IgG titer), were compared between qalb(BBB permeability) increased and normal groups. Complete measures were not obtained from 9 patients, but all other measures were included in the analysis.

Results: According to the calculated qalb, 15 patients with albumin quotient (qalb) $>(4+$ age/15) $\times 10-3$ were assigned to the qalb increased (high BBB permeability) group (33\%) and the remainder to the qalb normal group. Compared to the qalb normal group, the qalb increased group exhibited significantly lower serum albumin $(\mathrm{P}=0.001)$ and $\mathrm{CD} 4+\mathrm{T}$ cell count $(\mathrm{P}=0.044), \mathrm{CD} 8+\mathrm{T}$ cell count $(\mathrm{P}=0.014)$, and total $\mathrm{T}$ lymphocyte count $(\mathrm{P}=0.016)$. The qalb increased group proved higher $\mathrm{CSF}$ albumin, total protein, leukocyte count, and IgG titer (all $\mathrm{P}=0.000$ ). Optic neuritis and optic nerve abnormalities on magnetic resonance images were also more frequent in the qalb increased group ( $\mathrm{P}=0.037$ and 0.038 , respectively). Patients in the qalb increased group showed significantly poorer treatment response as indicated by the lower posttreatment change in Expanded Disability Status Scale (EDSS) score compared to the qalb normal group.

Conclusions: BBB permeability is strongly associated with the clinical features and treatment response of newly diagnosed NMOSD. The qalb is a potentially valuable indicator of disease severity and an index to guide personalized treatment.

Keywords: Blood-brain barrier (BBB); neuromyelitis optic spectrum disorders; newly diagnosis; optic neuritis; albumin quotient (qalb)

Submitted Nov 19, 2019. Accepted for publication Dec 27, 2019.

doi: 10.21037/apm.2019.12.12

View this article at: http://dx.doi.org/10.21037/apm.2019.12.12 


\section{Introduction}

Aquaporin-4 antibody (AQP4-IgG) is a specific NMOSD marker and the discovery of AQP4-IgG in NMOSD patients is the first clue for pathogenesis of disease. Neuromyelitis optica spectrum disorder (NMOSD) is an autoimmune-mediated inflammatory demyelinating disease with relapsing-remitting tendency. Notably, AQP4-IgG may persist in serum for many years, even with the absence of symptoms (1); therefore, the pathogenesis of NMOSD is still not fully understood.

Adding onto this, It is currently believed that the central pathogenic event in NMOSD is the entry of AQP4-IgG into the CNS through a damaged blood-brain barrier (BBB), triggering complement activation and granulocyte infiltration leading to astrocyte death (2). At the cellular level, AQP4 is expressed in astrocyte foot processes, with particularly abundant expression in the optic nerve, spinal cord, brainstem, hypothalamus, and cerebral ventricles, where it serves to maintain water balance by regulating the circulation of intracellular and extracellular water (3).

In AQP4-IgG seropositive rats, disruption of the BBB using pulsed ultrasound-induced NMO-like lesions in the brain and spinal cord by allowing entry of serum IgG. Central nervous system (CNS) inflammation, demyelination, and astrocyte damage are the primary pathological manifestations of NMO (4), and aberrant BBB permeability is associated with greater Extended Dysfunction Status Scale (EDSS) score and spinal cord lesion length (5). Moreover, BBB permeability, as assessed by the qalb, is strongly related to the degree of disability and brain atrophy after MS onset (6). In NMO spectrum disorder (NMOSD) as well, albumin quotient (qalb) is associated with the degree of motor disability (7).

However, there have been few studies on NMOSD in the acute phase, so there is a dearth of clinical and prognostic data on this patient population. Given the importance of BBB permeability in NMOSD pathogenesis, a convenient and straightforward biomarker is needed to evaluate disease severity and treatment outcome, predict recurrence, and guide individualized treatment decisions in newly diagnosed NMOSD patients. Therefore, we compared multiple peripheral types of blood and cerebrospinal fluid (CSF) metrics, magnetic resonance imaging (MRI) signs, and EDSS scores between NMOSD patients with high or normal BBB permeability as assessed by the qalb. The primary aim of this investigation is to determine whether higher BBB permeability during the acute phase of
NMOSD is associated with greater symptom severity and lower post-treatment change.

\section{Methods}

\section{Ethics statements}

The Ethics Committee approved the study of the First Affiliated Hospital of Guangxi Medical University.

\section{Study population}

We included 46 NMOSD patients admitted to the Department of Neurology, First Affiliated Hospital of Guangxi Medical University from January 2017 to March 2019. The cohort included 4 males and 42 females ranging from 14 to 78 years old. Age at onset was $42.9 \pm 15.5$ years, and the average disease duration was $1.14 \pm 1.05$ months. Symptoms gradually increased following onset. All patients were diagnosed based on the 2015 NMOSD diagnostic criteria developed by the International Panel for NMO Diagnosis (IPND). All patients provided informed consent to blood and CSF sampling by venipuncture and lumbar puncture, respectively. Exclusion criteria were treatment with methylprednisolone, gamma globulin, or plasma replacement before hospitalization, or diseases such as myasthenia gravis, meningitis, brain abscess, and epilepsy that may affect qalb index and EDSS score. In this study, $\mathrm{BBB}$ permeability was assessed according to qalb $=(4+$ age/15) $\times 10^{-3}$, increased $\mathrm{BBB}$ permeability was defined as qalb $>\left(4+\right.$ Age/15) $\times 10^{-3}(8)$. And all tests and qalb index calculations were performed before treatment.

\section{Treatment}

All patients with newly diagnosed NMOSD were treated with methylprednisolone $1.0 \mathrm{~g} /$ day for 3 days.

\section{Expanded disability status scale score}

The EDSS score was used to assess the severity of symptoms before and after treatment. An experienced neurologist performed the entire evaluation procedure.

\section{MRI images}

All patients were examined by T1-weighted, T2-weighted, and T2-Flair imaging of the optic nerve, brain, cervical 
Table 1 Clinical features

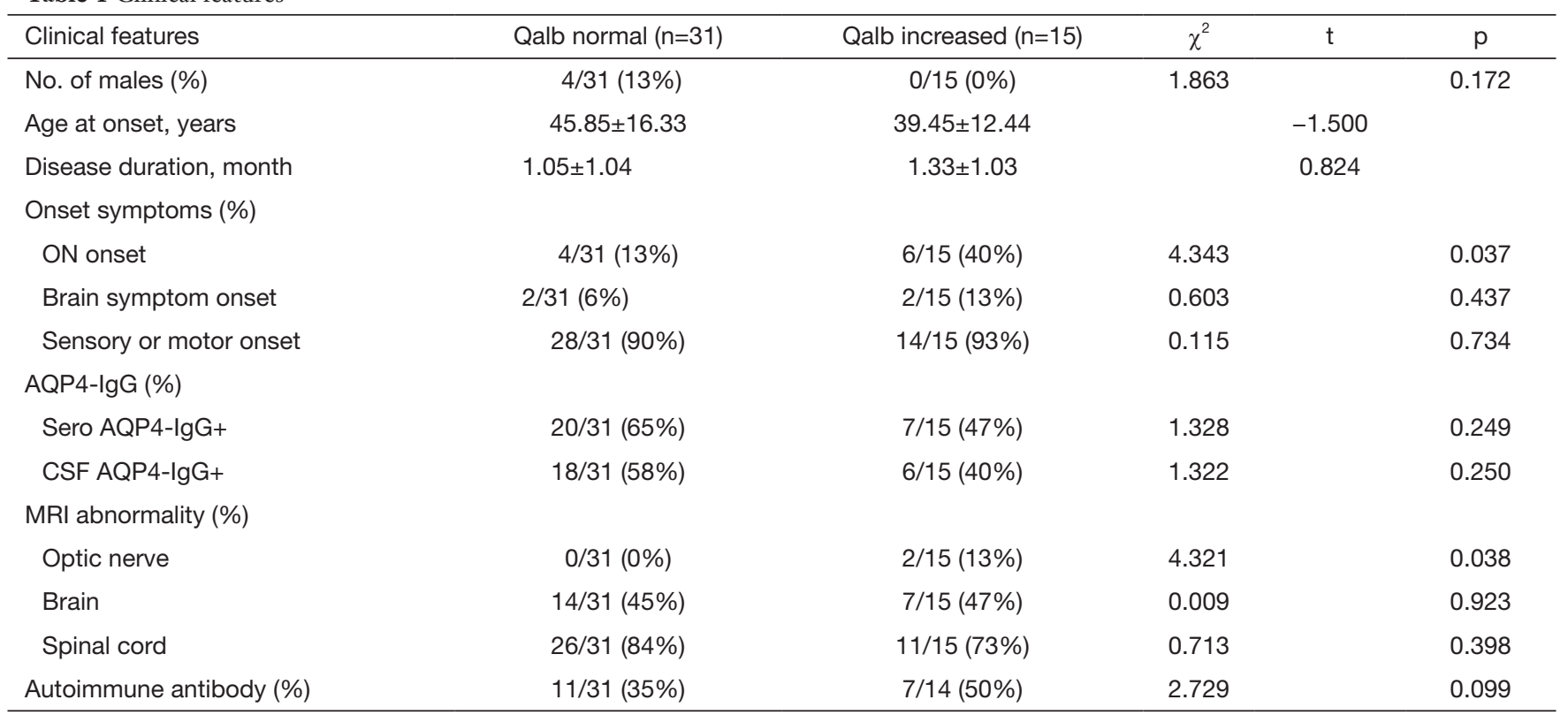

Qalb, albumin quotient; ON, optic neuritis.

spinal cord, and thoracic spinal cord using a $1.5-\mathrm{T}$ MRI system. An experienced neuroradiologist reviewed the images.

\section{AQP4-IgG analysis}

According to the latest guidelines, AQP4-IgG was detected by indirect immunofluorescence based on cell transfection.

\section{Peripheral blood (PB) and CSF analysis}

Total leukocyte, neutrophil, CD4+ T cell, CD8+ T cell, and total $\mathrm{T}$ lymphocyte counts, as well as albumin, complement $\mathrm{C} 3$, complement $\mathrm{C} 4$, and $\mathrm{AQP} 4-\mathrm{IgG}$ titer, autoimmune antibody (ANA/SSA/SSB/Ro-52) concentrations, were measured from serum samples. In addition, total leukocyte count and total protein, albumin, and AQP4$\mathrm{IgG}$ concentrations were measured from CSF samples. Measures of CD4+ T cells, CD8+ T cells, complement C3, and $\mathrm{C} 4$, and ANA/SSA/SSB/Ro-52 were not all conducted in nine patients, but all other measures were included in the analysis.

\section{Statistical analysis}

All statistical analyses were performed using SPSS
24.0 software. Categorical data are expressed as ratios and compared between groups by the chi-square test. Continuous data with normal distributions are expressed as $\bar{\chi} \pm S$ and compared between groups by independent samples $t$-test. Continuous data with non-normal distributions are expressed as the median [interquartile range] and compared between groups by Mann-Whitney U-test. A $\mathrm{P}<0.05$ (twotailed) is considered significant for all tests.

\section{Results}

\section{Clinical features}

Patients were divided into high and normal BBB permeability groups according to the qalb, with $15 / 46$ patients $(33 \%)$ in the qalb increased group [qalb > $(4+$ age $\left./ 15) \times 10^{-3}\right]$ and the remainder in the qalb normal group $\left[\mathrm{qalb} \leq(4+\right.$ age $\left./ 15) \times 10^{-3}\right]$ (Table 1$)$.

\section{Increased BBB permeability increased the probability of optic neuritis}

The probability of patients with optic neuritis was significantly higher in the qalb increased group $(\mathrm{P}=0.037)$. Similarly, the probability of patients with abnormal optic nerve $M R$ imaging manifestations on $M R$ images was higher in the qalb increased group $(\mathrm{P}=0.038)$ (Figure 1). 

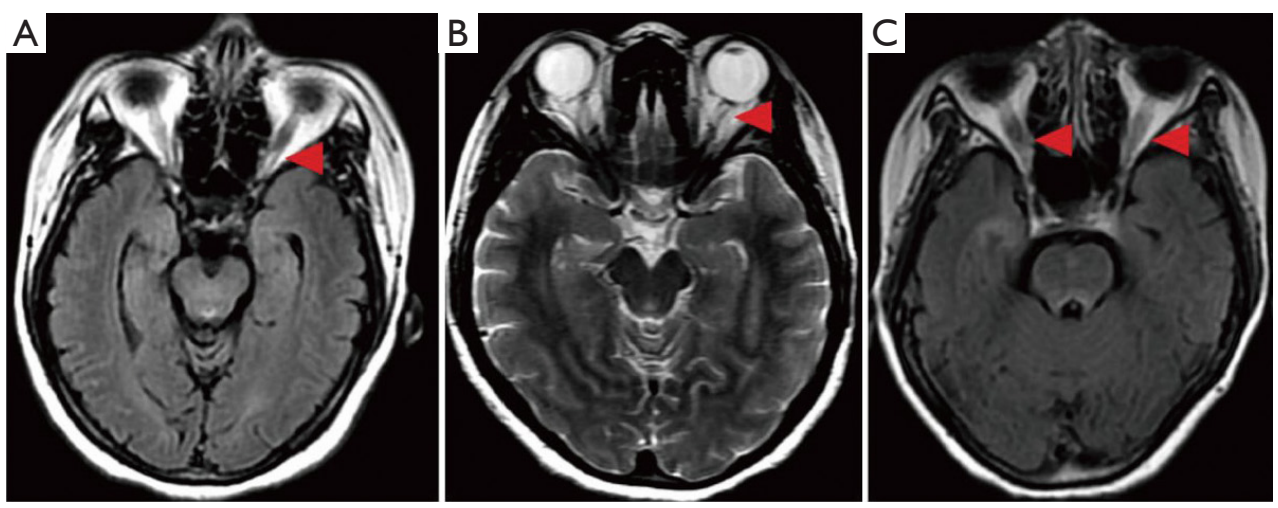

Figure 1 Abnormal optic nerves MR images in the qalb increased group. (A,B) Abnormal signal of the left nerve, (C) bilateral optic nerves thickening. MR, magnetic resonance; Qalb, albumin quotient.
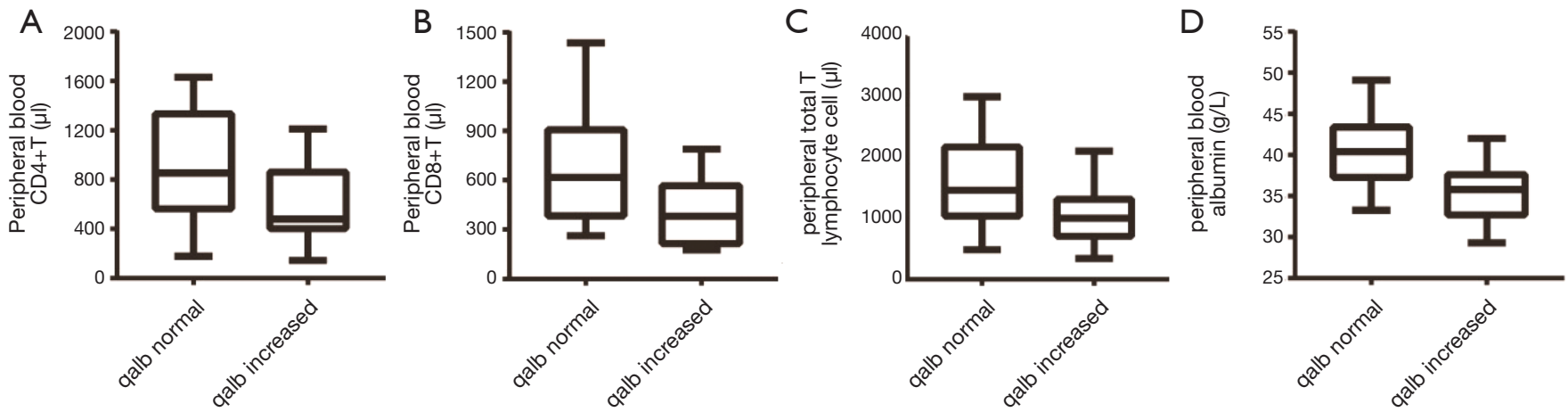

Figure 2 CD4+T/CD8+T/peripheral blood total $\mathrm{T}$ lymphocytes/peripheral blood albumin were lower in the qalb increased group than in the qalb normal group $(\mathrm{P}=0.044,0.014,0.016,0.001)$. Qalb, albumin quotient.

\section{$B B B$ permeability and $P B$ abnormalities}

Compared to the qalb normal group, the qalb increased group demonstrated no significant differences in total leukocyte count $(\mathrm{P}=0.200)$, neutrophil count $(\mathrm{P}=0.761)$, total lymphocyte count $(\mathrm{P}=0.679)$, complement $\mathrm{C} 3$ concentration $(\mathrm{P}=0.705)$, and complement $\mathrm{C} 4$ concentration $(\mathrm{P}=0.306)$. However, the qalb increased group exhibited significantly lower serum albumin $(\mathrm{P}=0.001)$ and $\mathrm{CD} 4+\mathrm{T}$ cell count $(\mathrm{P}=0.044), \mathrm{CD} 8+\mathrm{T}$ cell count $(\mathrm{P}=0.014)$, and total $\mathrm{T}$ lymphocyte count $(\mathrm{P}=0.016)$ (Figure 2).

\section{BBB permeability and CSF abnormalities}

The total number of leukocytes in the CSF was significantly elevated in the qalb increased group $(\mathrm{P}=0.007)$. Similarly, the qalb increased group demonstrated elevated CSF albumin, IgG, and total protein (all $\mathrm{P}=0.000$ ) (Figure 3).

\section{BBB permeability was associated with poorer treatment response}

Patients in the qalb normal group showed better symptom relief following methylprednisolone treatment than the qalb increased group, as indicated by the change in the EDSS score $(\mathrm{P}=0.028)$ (Table 2).

\section{Discussion}

While several previous studies have explored the relationship between qalb and NMOSD's clinical characteristics, it is unclear the effect of qalb on newly diagnosed NMOSD. We found that BBB disruption, as evidenced by high qalb, is associated with optic nerve 

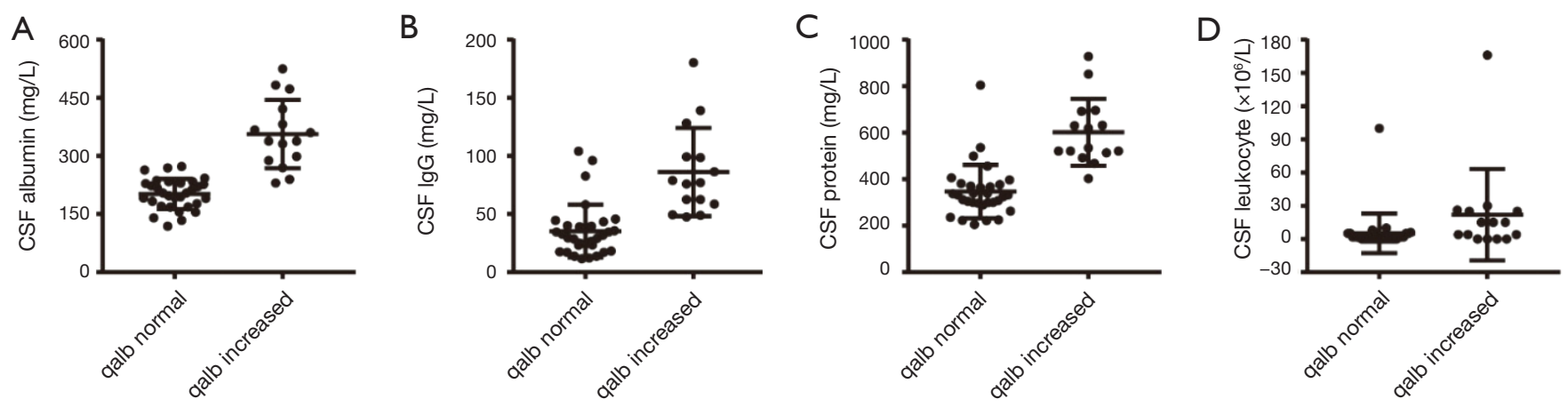

Figure $3 \mathrm{CSF}$ albumin/IgG/protein/leukocyte were higher in the qalb increased group than in the qalb normal group $(\mathrm{P}=0.000,0.000,0.000$, 0.007). CSF, cerebrospinal fluid; Qalb, albumin quotient.

Table 2 EDSS scale (before and after treatment)

\begin{tabular}{lcc}
\hline EDSS scale & Qalb normal $(n=31)$ & Qalb increased $(n=15)$ \\
\hline EDSS scale before treatment & $4.11 \pm 2.20$ & $3.70 \pm 2.27$ \\
EDSS scale after treatment & $3.39 \pm 2.38$ & $3.47 \pm 2.45$ \\
EDSS D-value & $0.73 \pm 0.84$ & $0.23 \pm 0.56$ \\
\hline
\end{tabular}

EDSS, Expanded Disability Status Scale; Qalb, albumin quotient; D-value, difference value.

abnormalities on MR images, poorer treatment response, lower CD4+ and CD8+ T cells in peripheral blood, and elevated total protein and leukocytes in CSF. Collectively, these blood and CSF changes are consistent with increased BBB permeability as a significant factor influencing NMOSD severity and treatment response in the acute phase.

Characteristics of the CNS microvasculature confer the $\mathrm{BBB}$, and under normal circumstances, provides a continuous barrier against peripheral cells (such as leukocytes), proteins, and other factors. Furthermore, BBB component cells express multiple transport pathways that can regulate the exchange of materials between the $\mathrm{PB}$ and the CNS. This selective barrier function is critical for maintaining the optimal extracellular microenvironment for neuronal signaling in the CNS. The BBB also protects the CNS from peripheral inflammatory factors and cells as well as viruses, bacteria, and bacterial toxins. Disruption of $\mathrm{BBB}$ integrity allows these factors, including immunocytes and pro-inflammatory cytokines, to access vulnerable neuronal populations, ultimately inducing neuroinflammatory diseases (9). Astrocytes are a critical component of the BBB and regulate barrier function (10). In addition, astrocytes couple neuronal activity to local blood flow thus mediating homeostatic regulation of neuronal metabolism (11). Astrocytes also promote angiogenesis by secreting angiopoietin 1 (Ang 1) and altering BBB permeability by regulating junctional protein expression (12). Aquaporin 4 (AQP4) is highly expressed in astrocyte foot processes that abut the neurovasculature, with particularly high expression in the brainstem, optic nerve, spinal cord, and periventricular regions (13), where it functions mainly to maintain water homeostasis. These regions of high AQP4 expressional are also the sites of typical NMO lesions (14), strongly implicating AQP4 in disease pathogenesis. Indeed, it is believed that under BBB degradation, AQP4IgG invades the CNS and binds to AQP4 on astrocytes, triggering an immune cascade that ultimately leads to astrocyte death, loss of extracellular and metabolic homeostasis, and further BBB degradation (13).

However, nearly one-third of NMO patients are seronegative for AQP4-IgG (14). Therefore, AQP4-IgG titer provides no prognostic information in these cases. Alternatively, our results suggest that qalb can be used for the prognosis of all NMOSD patients. We report that BBB disruption leads to worse prognosis in NMOSD, by allowing pro-inflammatory factors, leukocytes, and AQP4 antibodies to invade the retinal or CNS parenchyma, resulting in 
neuroinflammation. Our findings are in accord with previous reports that qalb is associated with CSF albumin, CSF leukocyte count, and spinal cord lesion length (5). In addition, patients with increased qalb demonstrated a higher probability of developing optic neuritis, a finding not previously reported. Retinal nerve fiber layer (RNFL) loss is more severe in NMO than in MS according to optical coherence tomography (OCT) (15). The onset of optic neuritis in NMOSD is believed to result from the entry of $\mathrm{T}$ cells into the CNS through the disrupted BBB. During optic neuritis, the retinal nerve fiber/ganglion cell layer often becomes thinner (16), suggesting damage to the optic nerve and retina (17). T cells can enter the retina through two pathways, the vascular pathway of the nerve fiber/ganglion cell/inner plexus layer or the outer nucleus/ outer plexus layer/core layer, AQP4-IgG, and complement resulting in the loss of AQP4 and destruction of Müller glial cells (18). This phenomenon has also been described in the autopsy results of NMOSD patients (19).

The mechanism of $\mathrm{CD} 4+\mathrm{T}$ and $\mathrm{CD} 8+\mathrm{T}$ cell reduction in PB of NMO is complex. OX40 (CD134), a member of the tumor necrosis factor (TNF) receptor family expressed primarily on activated CD4+ T and CD8 $+\mathrm{T}$ cells, was found to be downregulated in $\mathrm{PB}$ of NMO patients (20). Further, both the current results and a previous study (21) have found lower ratio PB CD4+ T cells and CD8+T cells, suggesting that $\mathrm{BBB}$ disruption is associated with a peripheral blood lymphocyte (PBL) imbalance. Based on earlier studies, we speculate that this reduction is related to the disruption of immune homeostasis. Linhares et al. (22) reported enhanced cell death in PHA-activated cultures from NMO patients and low release of interleukin-2 (IL-2) and interferon-gamma (INF- $\gamma$ ). This lowered release of IL-2 and IFN- $\gamma$ may trigger activation-induced cell death (AICD) of lymphocytes (23). Another potential mechanism is cell differentiation. Both T-helper cell 17 (Th17) and the cytokine IL-17A are reported to be abnormally increased in the $\mathrm{PB}$ of $\mathrm{NMO}$ patients. The resulting elevation in transforming growth factor- $\beta$, IL- 6 , and IL- 21 in $\mathrm{PB}$ promotes the differentiation of $\mathrm{CD} 4+\mathrm{T}$ cells and the secretion of cytokines. Abnormally high Th17 cell numbers elevated IL-17 secreted by CD8+ T cells, and higher IL-17A are all detected in NMO (24). In addition, Th17 cell number and IL-17A concentration are correlated with the EDSS score (25). However, we were unable to directly study this potential pathway due to an inability to detect CD4+ T cell subsets and related cytokines. Future studies are planned to assess this mechanism. Leukocytes were also significantly elevated in the CSF of patients with newly diagnosed NMOSD, especially the increased qalb group, in accord to a previous study by Wang et al. (5). We suspect that this phenomenon is related to cell migration.

Thus, the destruction of the BBB in NMOSD results in the immune invasion, which triggers the release of proinflammatory cytokines and complement factors from astrocytes resulting in further leukocytes infiltration (especially eosinophils and neutrophils) during the period of disease deterioration (14). This study also proves the utility of qalb as a biomarker for evaluating BBB permeability and for the prognosis of newly diagnosed NMOSD. Detection of high qalb may help guide individualized treatment.

There are limitations to this study. All patients have undergone only one lumbar puncture and only one qalb has been measured, while the effects of BBB permeability on NMOSD pathogenesis need to be confirmed by multiple lumbar punctures performed at different stages.

\section{Acknowledgments}

We want to thank Professor Yulan Tang and Shangling Pan for helpful guidance and the participating patients.

Funding: Guangxi Health Committee of China (S2019093). Guangxi Natural Science Foundation Grant (0991009, 2012GXNSFAA053082). National Natural Science Foundation of China Grant (81460194).

\section{Footnote}

Conflicts of Interest: All authors have completed the ICMJE uniform disclosure form and declare: The authors have no conflicts of interest to declare.

Ethical Statement: The authors are accountable for all aspects of the work in ensuring that questions related to the accuracy or integrity of any part of the work are appropriately investigated and resolved. The Ethics Committee approved the study of the First Affiliated Hospital of Guangxi Medical University [No. 2019(KY-E-119)].

Open Access Statement: This is an Open Access article distributed in accordance with the Creative Commons Attribution-NonCommercial-NoDerivs 4.0 International License (CC BY-NC-ND 4.0), which permits the noncommercial replication and distribution of the article with the strict proviso that no changes or edits are made and the original work is properly cited (including links to both the 
formal publication through the relevant DOI and the license). See: https://creativecommons.org/licenses/by-nc-nd/4.0/.

\section{References}

1. Bradl M, Reindl M, Lassmann H. Mechanisms for lesion localization in neuromyelitis optica spectrum disorders. Curr Opin Neurol 2018;31:325-33.

2. Vincent T, Saikali P, R, Roth A, et al. Functional consequences of neuromyelitis optica-IgG astrocyte interactions on blood-brain barrier permeability and granulocyte recruitment. J Immunol 2008;181:5730-7.

3. Papadopoulos MC, Verkman AS. Aquaporin 4 and neuromyelitis optica. Lancet Neurol 2012;11:535-44.

4. Yao X, Adams MS, Jones PD, et al. Noninvasive, Targeted Creation of Neuromyelitis Optica Pathology in AQP4IgG Seropositive Rats by Pulsed Focused Ultrasound. J Neuropathol Exp Neurol 2019;78:47-56.

5. Wang Y, Zhu M, Liu C, et al. Blood Brain Barrier Permeability Could Be a Biomarker to Predict Severity of Neuromyelitis Optica Spectrum Disorders: A Retrospective Analysis. Front Neurol 2018;9:648.

6. Uher T, Horakova D, Tyblova M, et al. Increased albumin quotient (QAlb) in patients after first clinical event suggestive of multiple sclerosis is associated with development of brain atrophy and greater disability 48 months later. Mult Scler 2016;22:770-81.

7. Tomizawa Y, Yokoyama K, Saiki S, et al. Blood-brain barrier disruption is more severe in neuromyelitis optica than in multiple sclerosis and correlates with clinical disability. J Int Med Res 2012;40:1483-91.

8. Reiber H, Otto M, Trendelenburg C, et al. Reporting cerebrospinal fluid data: knowledge base and interpretation software. Clin Chem Lab Med 2001;39:324-32.

9. Daneman R, Prat A. The Blood-Brain Barrier. Cold Spring Harbor Perspectives in Biology 2015;7:a020412.

10. Janzer RC, Raff MC. Astrocytes induce bloodbrain barrier properties in endothelial cells. Nature 1987;325:253-7.

11. Attwell D, Buchan AM, Charpak S, et al. Glial and neuronal control of brain blood flow. Nature 2010;468:232-43.

12. Prat A, Biernacki K, Wosik K, et al. Glial cell influence on the human blood-brain barrier. Glia 2001;36:145-55.

13. Wingerchuk DM. Neuromyelitis optica spectrum disorders. Continuum (Minneap Minn) 2010;16:105-21.

14. Wingerchuk DM, Lennon VA, Lucchinetti CF, et al. The spectrum of neuromyelitis optica. Lancet Neurol
2007;6:805-15.

15. Levin MH, Bennett JL, Verkman AS. Optic neuritis in neuromyelitis optica. Prog Retin Eye Res 2013;36:159-71.

16. Bennett JL, de Seze J, Lana-Peixoto M, et al. Neuromyelitis optica and multiple sclerosis: Seeing differences through optical coherence tomography. Mult Scler 2015;21:678-88.

17. Zeka B, Hastermann M, Kaufmann N, et al. Aquaporin 4-specific T cells and NMO-IgG cause primary retinal damage in experimental NMO/SD. Acta Neuropathol Commun 2016;4:82.

18. Zeka B, Lassmann H, Bradl M. Muller cells and retinal axons can be primary targets in experimental neuromyelitis optica spectrum disorder. Clin Exp Neuroimmunol 2017;8:3-7.

19. Hokari M, Yokoseki A, Arakawa M, et al. Clinicopathological features in anterior visual pathway in neuromyelitis optica. Ann Neurol 2016;79:605-24.

20. Alidadiani P, Eskandari N, Shaygannejad V, et al. Expression of OX40 Gene and its Serum Levels in Neuromyelitis Optica Patients. Biomol Concepts 2019;10:62-7.

21. Uzawa A, Mori M, Hayakawa S, et al. Expression of chemokine receptors on peripheral blood lymphocytes in multiple sclerosis and neuromyelitis optica. BMC Neurol 2010;10:113.

22. Linhares UC, Schiavoni PB, Barros PO, et al. The ex vivo production of IL-6 and IL-21 by CD4+ T cells is directly associated with neurological disability in neuromyelitis optica patients. J Clin Immunol 2013;33:179-89.

23. Akkoc T, de Koning PJ, Ruckert B, et al. Increased activation-induced cell death of high IFN-gammaproducing $\mathrm{T}(\mathrm{H}) 1$ cells as a mechanism of $\mathrm{T}(\mathrm{H}) 2$ predominance in atopic diseases. J Allergy Clin Immunol 2008;121:652-8.e1.

24. Wang HH, Dai YQ, Qiu W, et al. Interleukin-17-secreting $\mathrm{T}$ cells in neuromyelitis optica and multiple sclerosis during relapse. J Clin Neurosci 2011;18:1313-7.

25. Li Y, Wang H, Long Y, et al. Increased memory Th17 cells in patients with neuromyelitis optica and multiple sclerosis. J Neuroimmunol 2011;234:155-60.

Cite this article as: Liang S, Qin Q, Tang Y, Liao W, Yang Y, He J, Li L. Impact of blood-brain barrier disruption on newly diagnosed neuromyelitis optica spectrum disorder symptoms and prognosis. Ann Palliat Med 2020;9(2):324-330. doi: 10.21037/apm.2019.12.12 